\section{P115 SEXUALISED DRUG USE IN PATIENTS ATTENDING AN NHS WALK-IN CENTRE}

${ }^{1}$ Jodie Scrivener*, 'Tamuka Gonah, 'Isata Gando, 'Rageni Sangha, ${ }^{2}$ Daniel Richardson. ${ }^{1}$ Care UK, Brighton, UK; ${ }^{2}$ Brighton \& Sussex University Hospitals NHS Trust, Brighton, UK

10.1136/sextrans-2015-052126.158

Background/introduction Recent increases in reported recreational-drug use in MSM are associated with high-risk sexual behaviour and poor (sexual) health outcomes. There is little comparative research in non-MSM. Brighton Station Health Centre offers both walk-in general and sexual-health services.

Aim(s)/objectives To compare reported drug use between patients attending the sexual health and general walk-in services. Methods A Self-completed service-evaluation recording details of drug use in the previous 6-months was offered to all patients attending during a week in October-2014.

Results 125/633(19\%) completed the survey; 75/125(60\%) were attending sexual-health; 33/125 identified as male and 1 as trans*. 8/33(24\%) men identified as MSM. The median age was 30 -years. $12 / 50(24 \%)$ patients attending general-health and 18 (24\%) sexual-health reported drug use; most respondents using $>1$ drug: MDMA $(\mathrm{n}=21)$ and Cocaine $(\mathrm{n}=18)$ most popular. Ketamine was reported by 7/125-all heterosexual; Mephedrone, GBL and Crystal almost exclusively by MSM. 1/125 (MSM) reported Intravenous-Drug use (Crystal-Meth). Most were infrequent drug-users with $21 / 125(70 \%)$ using $<1$ /month. $2 / 125(6 \%)$ reported using drugs $>1$ /week. $22 / 30(73 \%)$ did not feel that drugs were having a negative effect on their life; 19/30 63\% said that their risk behaviour and likelihood of having unprotected sex was not increased using drugs. Only 3 patients wanted advice on drug-use. Most (46\%) would prefer advice about drugs from specialist drug services.

Discussion/conclusion In this small study there was no difference in reported drug use between MSM and non MSM, however the pattern of drug-use differs. As most felt that their druguse was not problematic they are unlikely to seek advice and so general walk-in services offer opportunities for early intervention.

\section{P116 AN AUDIT OF GONORRHOEA MANAGEMENT IN A UK SEXUAL HEALTH CLINIC}

Karan Bhatt*, Lisa Goodall. SSOTP NHS Trust, Stoke-on-Trent, Staffordshire, UK

10.1136/sextrans-2015-052126.159

Background/introduction Gonorrhoea (GC) is a prevalent sexually-transmitted infection in the UK. The British Society for Sexual Health and HIV (BASHH) published guidelines in 2011 for the management of GC.

Aim(s)/objectives To audit the management of all patients who tested positive for GC at our centre between 1 May 2013 and 1 May 2014 against BASHH guidelines.

Methods A proforma was developed and a retrospective notes review performed for all patients who tested positive for GC from 1 May 2013 to 1 May 2014.

Results 115 patients tested positive for GC during the audit period. The prevalence of GC in this cohort was $0.9 \% .46 \%$ of patients had symptom (s), 54\% were truly asymptomatic, $18 \%$ of patients presented as contacts. Microscopy was performed on $80 \%$ of symptomatic patients and intracellular gram-negative diplococci were seen in $66 \%$ of these. $97 \%$ of patients were diagnosed by PCR testing and one by culture. 2 patients were diagnosed elsewhere with negative testing at our clinic. $83 \%$ had GC cultures prior to treatment. The sensitivity of culture compared to PCR testing was 59\%. 93\% of patients were treated as per BASHH guidelines. $65 \%$ were documented to have received written information about their diagnosis. All patients were offered test of cure, of which $61 \%$ attended within 2 weeks. $92 \%$ of patients saw health advisers for partner notification.

Discussion/conclusion Management of GC was largely in line with BASHH guidance. However, this study highlighted a need to increase written information offer and to encourage attendance for test of cure.

\section{P117 A QUALITATIVE ASSESSMENT OF UK SEXUAL HEALTHCARE PROFESSIONALS' VIEWS ON TARGETED VACCINATION AGAINST HUMAN PAPILLOMAVIRUS (HPV) FOR MEN WHO HAVE SEX WITH MEN (MSM)}

${ }^{1}$ Tom Nadarzynski*, ${ }^{1}$ Carrie Llewellyn, ${ }^{2}$ Daniel Richardson, ${ }^{1}$ Alex Pollard, ${ }^{1}$ Helen Smith. ${ }^{1}$ Brighton and Sussex Medical School, Brighton, UK; ${ }^{2}$ Brighton and Sussex University Hospitals, Brighton, UK

\subsection{6/sextrans-2015-052126.160}

Background Female-only HPV vaccination will fail to protect MSM against HPV and its sequelae i.e. genital warts and anal cancers. In the absence of gender-neutral HPV vaccination, targeted vaccination for MSM at sexual health clinics offers a valuable preventive opportunity.

Aims To identify sexual healthcare professionals' (SHCPs) perceived barriers and facilitators for MSM-targeted HPV vaccination.

Methods Nineteen telephone interviews, with UK-based selfreferred SHCPs (13 doctors, 3 nurses, 3 health advisers), were conducted in October and November 2014. The interviews were recorded and transcribed verbatim. Data were analysed thematically by two researchers.

Results Nine themes were identified. The major perceived barriers were: 'concerns about vaccination programme equity and equality'; 'concerns about vaccination effectiveness'; 'challenges with targeting MSM'; 'obstacles with HPV vaccination delivery' and 'negative public reaction to targeting MSM'. The main facilitators were: 'policies and guidelines'; 'rising awareness'; 'acceptable settings' and 'adequate vaccination procedures'. While SHCPs expressed varied and sometimes contradictory views on MSM-targeted HPV vaccination, most agreed that HPV vaccination, inclusive of all school-aged boys, would be the most suitable strategy.

Conclusion Although SHCPs recognised a need to protect MSM against HPV, several challenges and obstacles associated with the introduction of MSM-targeted HPV vaccination in the UK were reported. Solutions on individual, organisational and public levels were offered. SHCPs' perspectives and concerns need to be addressed when developing policies and guidelines for a potential MSM-targeted HPV vaccination. Future research needs to examine whether negative views of SHCPs towards MSMtargeted HPV vaccination are associated with lower HPV vaccine acceptability and uptake in MSM. 